\title{
Aminophosphonate Inhibitors of Dialkylglycine Decarboxylase: Structural Basis for Slow Binding Inhibition ${ }^{\dagger, *}$
}

\author{
Wenshe Liu, Claude J. Rogers, Andrew J. Fisher, and Michael D. Toney* \\ Department of Chemistry, University of California, Davis, California 95616 \\ Received June 18, 2002; Revised Manuscript Received August 5, 2002
}

\begin{abstract}
The kinetics of inhibition of dialkylglycine decarboxylase by five aminophosphonate inhibitors are presented. Two of these [(R)-1-amino-1-methylpropanephosphonate and $(S)$-1-aminoethanephosphonate $]$ are slow binding inhibitors. The inhibitors follow a mechanism in which a weak complex is rapidly formed, followed by slow isomerization to the tight complex. Here, the tight complexes are bound 10-fold more tightly than the weak, initial complexes. The slow onset inhibition occurs with $t_{1 / 2}$ values of 1.3 and 0.55 min at saturating inhibitor concentrations for the AMPP and $S$-AEP inhibitors, respectively, while dissociation of these inhibitor complexes occurs with $t_{1 / 2}$ values of 13 and $4.6 \mathrm{~min}$, respectively. The $\mathrm{X}$-ray structures of four of the inhibitors in complex with dialkylglycine decarboxylase have been determined to resolutions ranging from 2.6 to $2.0 \AA$, and refined to $R$-factors of $14.5-19.5 \%$. These structures show variation in the active site structure with inhibitor side chain size and slow binding character. It is proposed that the slow binding behavior originates in an isomerization from an initial complex in which the PLP pyridine nitrogen-D243 OD2 distance is $\sim 2.9 \AA$ to one in which it is $\sim 2.7 \AA$. The angles that the $\mathrm{C}-\mathrm{P}$ bonds make with the p orbitals of the aldimine $\pi$ system are correlated with the reactivities of the analogous amino acid substrates, suggesting a role for stereoelectronic effects in Schiff base reactivity.
\end{abstract}

Pyridoxal phosphate (PLP) ${ }^{1}$ dependent enzymes are ubiquitous in the nitrogen metabolism of all organisms, catalyzing a wide variety of reactions at the $\alpha-, \beta$-, and $\gamma$-carbons of amine and amino acid substrates $(1-3)$. One group of these catalyzes $\alpha$-decarboxylation of amino acids to yield amine products formed by replacement of the substrate carboxylate with a proton. Another class catalyzes the transfer of amino groups between amines or amino acids and $\alpha$-keto acids, in a reaction known as transamination. Dialkylglycine decarboxylase is an unusual enzyme that combines both decarboxylation and transamination half-reactions in its normal catalytic cycle (Scheme 1). This unusual combination requires that the decarboxylation reaction proceeds with transfer of the substrate amino group to the coenzyme to form the PMP enzyme as an intermediate in the catalytic cycle. This is effectively an oxidative decarboxylation with respect to the amino acid substrate, and is accomplished by

\footnotetext{
$\dagger$ This work was supported by Grant GM54779 to M.D.T. from the National Institutes of Health. W.L. is supported by Grant 2001-07 of the University of California Systemwide Biotechnology Research Program.

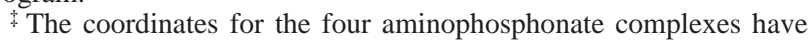
been deposited in the Protein Data Bank as the following entries: 1M0N for ACPP, $1 \mathrm{M} 0 \mathrm{O}$ for AMPP, $1 \mathrm{M} 0 \mathrm{P}$ for APEP, and 1M0Q for $S$-AEP.

* To whom correspondence should be addressed. E-mail: toney@ chem.ucdavis.edu. Telephone: (530) 754-5282. Fax: (530) 752-8995.

${ }^{1}$ Abbreviations: PLP, pyridoxal 5'-phosphate; PMP, pyridoxamine 5'-phosphate; DGD, 2,2-dialkylglycine decarboxylase; DGD-K, DGD with $\mathrm{K}^{+}$bound near the active site; DGD-Li, DGD with $\mathrm{Li}^{+}$bound near the active site; DGD-Rb, DGD with $\mathrm{Rb}^{+}$bound near the active site; DGD-Na, DGD with $\mathrm{Na}^{+}$bound near the active site; $2^{\circ} \mathrm{ADH}$, secondary alcohol dehydrogenase; TEA, triethanolamine; AIB, aminoisobutyrate; MES, morpholinoethanesulfonate; AEP, 1-aminoethanephosphonate; AMPP, 1-amino-1-methylpropanephosphonate; ACPP, 1-aminocyclopentanephosphonate; APEP, 1-amino-1-phenylethanephosphonate.
}

Scheme 1: Half-Reactions Catalyzed by Dialkylglycine Decarboxylase

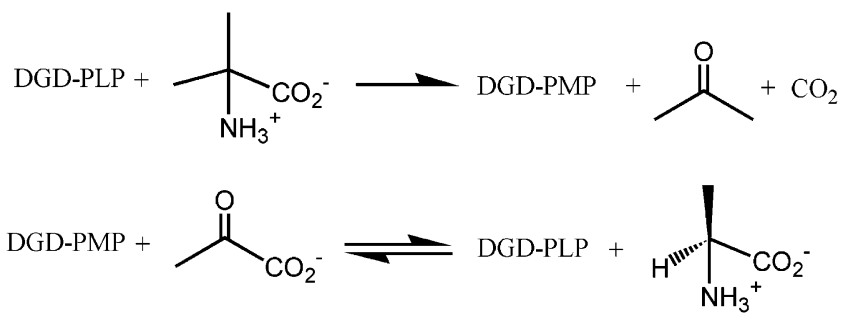

specific protonation of coenzyme $\mathrm{C}^{\prime}$ ' instead of replacing the substrate carboxylate with a proton. Zhou et al. (4) demonstrated that the $\mathrm{C}-\mathrm{C}$ bond breaking at $\mathrm{C} \alpha$ and the $\mathrm{C}-\mathrm{H}$ bond making at $\mathrm{C}^{\prime}$ occur simultaneously via a concerted transition state, thus achieving extraordinarily high specificity for oxidative decarboxylation.

In 1966, Dunathan (5) proposed that PLP dependent enzymes can control reaction specificity through stereoelectronic effects. This would occur by aligning the scissile bond such that it is parallel to the p orbitals of the conjugated $\pi$ system, thereby giving maximal transition state orbital overlap and maximal resonance stabilization. The unusual reaction specificity of DGD allowed Sun et al. (6) to use alternate substrates to demonstrate qualitatively the role of stereoelectronic effects in control of reaction specificity. This analysis employed a functional model of the active site with three binding subsites whose locations and specificities have been described $(6,7)$. These are subsite A, near Q52 and $\mathrm{K} 272$, which is the locus of bond making and breaking; subsite B, near R406 and S215; and subsite C, near M141. Two carboxylate-containing inhibitor structures were previously reported $(8)$, showing the carboxylate groups bound 


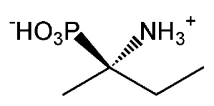

(R)-1-amino-1-methylpropanephosphonate (AMPP)

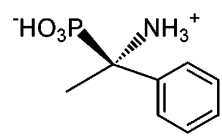

(R)-1-amino-1-phenylethanephosphonate (APEP)

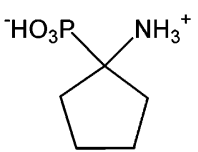

1-aminocyclopentanephosphonate (ACPP)

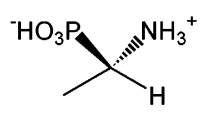

(S)-1-aminoethanephosphonate (S-AEP)

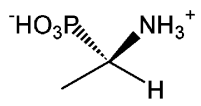

(R)-1-aminoethanephosphonate (R-AEP)

FIGURE 1: Structures, names, and abbreviations for the aminophosphonate inhibitors used in this study.

in subsite B. The phosphonate groups of the aminophosphonate inhibitors employed here were expected to bind in subsite A, where decarboxylation of $\alpha$-amino acid substrates occurs. This would have provided a clear picture of the interactions made by substrate carboxylates in their catalytically productive conformation. It is found that the phosphonate groups are bound in a location between what are considered the A and B subsites.

The kinetics of inhibition by the aminophosphonate inhibitors are also presented. Two of the five that were analyzed are slow binding inhibitors. These act via a weak enzyme-inhibitor complex, followed by slow isomerization to the final, tight complex. The dissociation constants for the initial weak complexes of the tight binding and for the complexes of the normally behaving inhibitors are close to the values of the corresponding amino acid substrates. The slow binding behavior is proposed to arise from an isomerization of the PLP-inhibitor aldimine geometry from an initial state in which the PLP pyridine nitrogen-D243 OD2 distance is $\sim 2.9 \AA$ to one in which it is shortened to $\sim 2.7$ A.

\section{EXPERIMENTAL PROCEDURES}

Materials. Succinic acid and TEA were purchased from Fisher. PLP was obtained from Aldrich. NADPH was from Roche. AIB, pyruvic acid, $2^{\circ} \mathrm{ADH}$, and $R$ - and $S$-AEP were purchased from Aldrich. PEG4000 and sodium pyruvate were from Fluka. MES was from Research Organics. AMPP, ACPP, and APEP (Figure 1) were synthesized according to a literature procedure (9). The racemic mixtures of AMPP and APEP were used without resolution, and the reported $K_{\mathrm{i}}$ values are the observed values divided by 2 , since no evidence for binding of both isomers was observed.

Steady State Kinetic Assay of DGD. AIB decarboxylation was followed by coupling acetone produced from AIB to the $2^{\circ} \mathrm{ADH}$ reaction as described previously (6). A Kontron UVIKON 9420 spectrometer was employed to monitor the decrease in NADPH absorbance at $340 \mathrm{~nm}$ that occurs during reduction of acetone to 2-propanol. Pyruvate was used as the $\alpha$-keto acid substrate for transamination in all assays.

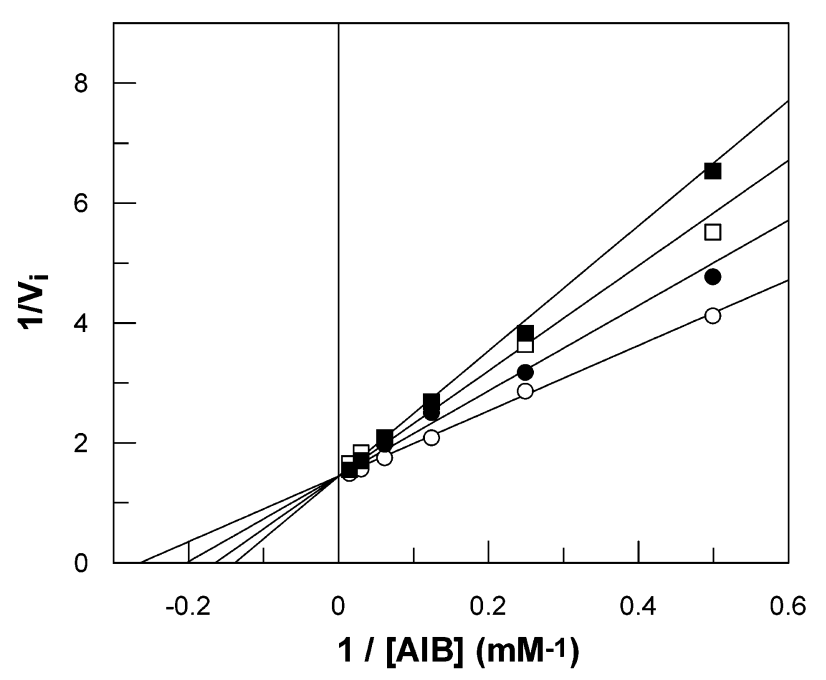

FIGURE 2: Double-reciprocal plot for inhibition of DGD by AMPP. The concentrations of the racemic mixture that were used are 0 $(\bigcirc), 3(\bullet), 6(\square)$, and $9 \mathrm{mM}(\boldsymbol{\square})$. The lines are results of global fitting of the data set to the competitive inhibition equation (eq 1). The initial rates employed here were taken from the beginnings of the progress curves before the onset of slow binding inhibition.

The reaction mixtures contained $100 \mathrm{mM}$ TEA $\cdot$ succinate $(\mathrm{pH}$ 7.8), $100 \mathrm{mM}$ dipotassium succinate, $2 \mathrm{mM}$ sodium pyruvate, 1 unit/mL $2^{\circ} \mathrm{ADH}, 50 \mu \mathrm{M}$ PLP, $300 \mu \mathrm{M}$ NADPH, $0.1 \mu \mathrm{M}$ DGD, 2-64 mM AIB, and various concentrations of inhibitors.

Inhibition Kinetics. Initial rates for six concentrations of 2-aminoisobutyrate at each of four concentrations of AMPP were measured to characterize the inhibition mechanism. AMPP is a slow binding inhibitor, as described below. Characterization of the inhibition mechanism employed the initial rates measured before the onset of slow binding inhibition. The Lineweaver-Burke plot for these data is presented in Figure 2, and clearly indicates competitive inhibition. The data were fitted to eq 1 , which describes competitive inhibition, to obtain the $K_{\mathrm{i}}$ value.

$$
v_{\mathrm{i}}=\frac{V_{\max }[\mathrm{AIB}]}{K_{\mathrm{M}}\left(1+\frac{[\mathrm{II}]}{K_{\mathrm{i}}}\right)+[\mathrm{AIB}]}
$$

Here, $v_{\mathrm{i}}$ is the initial rate, $V_{\max }$ the maximal rate, $K_{\mathrm{m}}$ the Michaelis constant, I the AMPP inhibitor, and $K_{\mathrm{i}}$ the inhibition constant.

The inhibition mechanisms for the other four aminophosphonate inhibitors were assumed to be competitive on the basis of the results with AMPP. Thus, the $K_{\mathrm{i}}$ values for these were obtained by holding the concentration of AIB constant and varying the inhibitor concentration. These data were employed with eq 1 , fixing $K_{\mathrm{M}}$ at its known value from the experiment with AMPP.

Determination of $K_{i}^{*}$. AMPP and $S$-AEP are slow binding inhibitors of DGD. The overall dissociation constant, $K_{\mathrm{i}}^{*}$ (10), for these inhibitors was measured by preincubation of DGD in a $1 \mathrm{~mL}$ solution containing $100 \mathrm{mM}$ TEA $\cdot$ succinate ( $\mathrm{pH}$ 7.8), $100 \mathrm{mM}$ dipotassium succinate, $2 \mathrm{mM}$ sodium pyruvate, 1 unit/mL $2^{\circ} \mathrm{ADH}, 50 \mathrm{mM}$ PLP, $300 \mu \mathrm{M}$ NADPH, and various inhibitor concentrations for $30 \mathrm{~min}$. This preincubated solution was then mixed with $20 \mu \mathrm{L}$ of $1 \mathrm{M}$ 
Scheme 2: Mechanism of Slow Binding Competitive Inhibition

$$
\mathrm{K}_{\mathrm{i}}^{*}\left\{\begin{array}{l}
\mathrm{E}+\mathrm{S} \stackrel{\mathrm{K}_{\mathrm{M}}}{\longrightarrow} \mathrm{ES} \stackrel{\mathrm{k}_{\mathrm{cat}}}{\longrightarrow} \mathrm{E}+\mathrm{P} \\
\mathrm{I} \\
\| \mathrm{K}_{\mathrm{i}} \\
\mathrm{EI} \\
\mathrm{k}_{2} \|_{\mathrm{k}_{1}} \\
\mathrm{EI}^{*}
\end{array}\right.
$$

$\mathrm{AIB}$, and the decrease in NADPH absorbance at $340 \mathrm{~nm}$ was monitored. This preincubation time was determined to be sufficient for full equilibration of inhibitor binding; thus, the measured rates reflect the slow binding inhibition of DGD. The value of $K_{\mathrm{i}}^{*}$ was determined by fitting initial rates to eq 2 :

$$
v_{\mathrm{i}}=\frac{V_{\max }[\mathrm{AIB}]}{K_{\mathrm{M}}\left(1+\frac{[\mathrm{I}]}{K_{\mathrm{i}}}\right)+[\mathrm{AIB}]} \frac{1+\alpha[\mathrm{I}] / K_{\mathrm{i}}}{1+\alpha[\mathrm{I}] / K_{\mathrm{i}}^{*}}
$$

where $K_{\mathrm{i}}^{*}$ is the slow binding dissociation constant and $\alpha$ is the ratio of the final reaction volume to the volume of the preincubation mixture (i.e., 0.98).

Measurement of Slow Binding Isomerization Rate Constants. Scheme 2 shows the kinetic mechanism for competitive inhibition with slow binding of the inhibitor. To measure $k_{2}$, DGD was incubated with $50 \mathrm{mM}$ TEA $\cdot$ succinate ( $\mathrm{pH} 7.8$ ), $50 \mathrm{mM}$ dipotassium succinate, $20 \mu \mathrm{M}$ PLP, and $8 \mathrm{mM}$ AMPP or $2 \mathrm{mM} S$-AEP for $1 \mathrm{~h}$. Then, $20 \mu \mathrm{L}$ of this preincubated DGD solution was mixed with $1 \mathrm{~mL}$ of a cocktail containing $100 \mathrm{mM}$ TEA $\cdot$ succinate $(\mathrm{pH} 7.8), 100$ $\mathrm{mM}$ dipotassium succinate, $2 \mathrm{mM}$ sodium pyruvate, 1 unit/ $\mathrm{mL} 2^{\circ} \mathrm{ADH}, 50 \mu \mathrm{M}$ PLP, $300 \mu \mathrm{M} \mathrm{NADPH}$, and 25, 50, or $100 \mathrm{mM}$ AIB, and the reactions were monitored at $340 \mathrm{~nm}$. The ratio of the final inhibitor concentration to that of AIB was very small, thus enabling simple measurement of $k_{2}$ in Scheme 2 by enforcing irreversible conditions on the dissociation of the inhibitor. The reaction curves were fitted to eq 3

$$
A_{340}=A_{340}^{0}-\left[v_{\mathrm{f}} t+\frac{\left(v_{\mathrm{i}}-v_{\mathrm{f}}\right)\left(1-\mathrm{e}^{-k t}\right)}{k}\right]
$$

where $A_{340}$ is the NADPH absorbance at $340 \mathrm{~nm}, v_{\mathrm{i}}$ the initial reaction rate, $v_{\mathrm{f}}$ the final reaction rate, and $k$ the apparent isomerization rate constant. The values of $k_{1}$ were determined using eq 4 , and the values of $k_{2}, K_{\mathrm{i}}$, and $K_{\mathrm{i}}^{*}$ as determined above.

$$
K_{\mathrm{i}}^{*}=K_{\mathrm{i}} \frac{k_{2}}{k_{1}+k_{2}}
$$

Spectral Kinetic Analysis of Isomerization of DGD-AMPP Complexes. The spectral changes in the coenzyme on reaction of DGD-PLP with AMPP were used to determine independently the values of $k_{1}$ and $k_{2}$ for AMPP. DGD-PLP, $4 \mathrm{mg} /$ $\mathrm{mL}$ in $100 \mathrm{mM}$ TEA $\cdot$ succinate, $100 \mathrm{mM}$ dipotassium succinate, and $5 \mu \mathrm{M}$ PLP, was mixed with various concen-

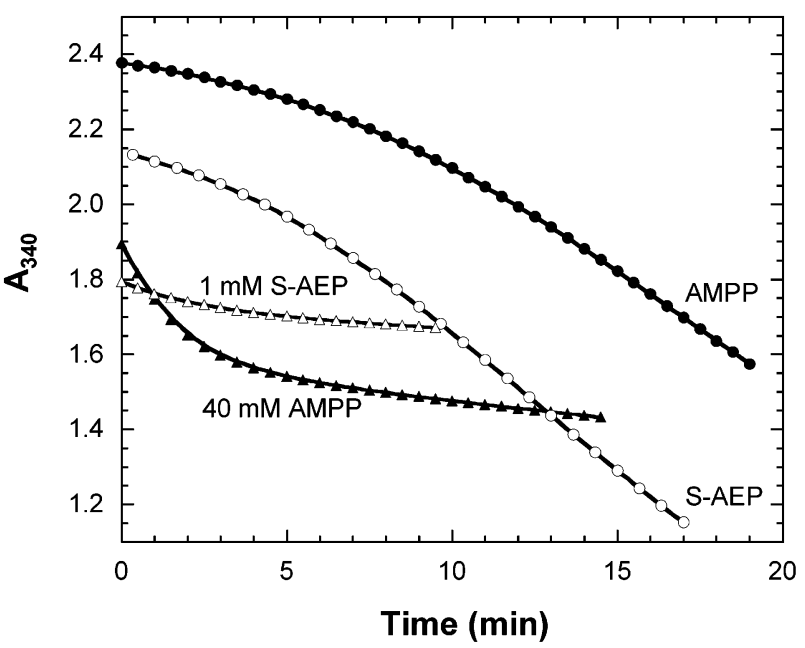

FIGURE 3: Kinetics of slow binding inhibition by AMPP and $S$-AEP. The triangles are for data from experiments in which the reaction mixtures contained the specified concentration of inhibitor and the reactions were initiated by addition of DGD. The circles are for data from experiments in which the DGD-inhibitor complex was preformed and diluted into a reaction mixture without inhibitor and with a high AIB concentration (100 mM).

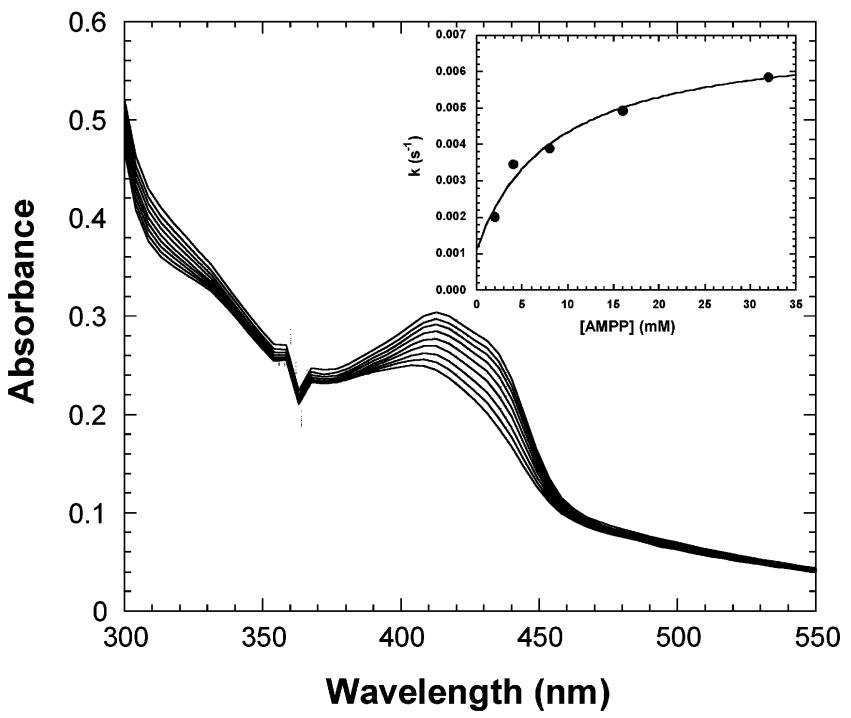

FIGURE 4: Spectral kinetic analysis of the binding of AMPP to DGD-PLP. The absorbance spectra that are shown were collected using a Hewlett-Packard 8453 diode-array spectrometer over a period of $10 \mathrm{~min}$ for the reaction of $2 \mathrm{mg} / \mathrm{mL}$ DGD-PLP with 32 mM AMPP (total concentration of the racemic mixture). The absorbance increases with time over the entire spectral range. The inset shows the observed pseudo-first-order rate constant as a function of the total concentration of the racemic mixture.

trations of AMPP in $100 \mathrm{mM}$ TEA $\cdot$ succinate, $100 \mathrm{mM}$ dipotassium succinate, and $5 \mu \mathrm{M}$ PLP at $25^{\circ} \mathrm{C}$. The spectral changes were globally fitted to a single-exponential kinetic function using the program SPECFIT to obtain apparent rate constants. The data are shown in Figure 4. The apparent rate constants were used, along with the known $K_{\mathrm{i}}$ value, in a least-squares fit to eq 5 to obtain values of $k_{1}$ and $k_{2}$.

$$
k_{\mathrm{app}}=k_{2}+k_{1} \frac{[\mathrm{AMPP}]}{K_{\mathrm{i}}+[\mathrm{AMPP}]}
$$

Crystallization, X-ray Diffraction Data Collection, and Structural Refinements. Crystals of DGD were obtained by 


\begin{tabular}{|c|c|c|c|c|c|c|c|}
\hline inhibitor & homologous substrate & $k_{\text {cat }}\left(\mathrm{s}^{-1}\right)$ & $K_{\mathrm{m}}(\mathrm{mM})$ & $K_{\mathrm{i}}^{c}(\mathrm{mM})$ & $K_{\mathrm{i}} * d(\mathrm{mM})$ & $k_{1}\left(\times 10^{3} \mathrm{~s}^{-1}\right)$ & $k_{2}\left(\times 10^{3} \mathrm{~s}^{-1}\right)$ \\
\hline APEP & L-phenylglycine ${ }^{g}$ & $0.20^{a}(0.03)$ & $8.3^{a}(2.2)$ & $13(3)$ & & & \\
\hline ACPP & aminocyclopentane carboxylate & $0.98^{a}(0.03)$ & $4.5^{a}(0.4)$ & $6.0(0.8)$ & & & \\
\hline AMPP & L-isovaline & $14^{b}$ & $25^{b}$ & $4.9(0.8)$ & $0.5(0.06)$ & $\begin{array}{l}7.9^{e}(0.9) \\
6.0^{f}(0.6)\end{array}$ & $\begin{array}{l}0.9^{e}(0.1) \\
1.3^{f}(0.3)\end{array}$ \\
\hline$R$-AEP & L-alanine & $0.033^{a}(0.003)$ & $7^{a}(1)$ & $19(1)$ & & & \\
\hline$S$-AEP & D-alanine & $0.20^{a}(0.02)$ & $7.1^{a}(1.5)$ & $1.1(0.1)$ & $0.13(0.01)$ & $19^{e}(1.4)$ & $2.5^{e}(0.2)$ \\
\hline
\end{tabular}

${ }^{a} k_{\text {cat }}$ and $K_{\mathrm{m}}$ of homologous substrates are from ref 6 and are for the decarboxylation of the specified substrates. ${ }^{b} k_{\text {cat }}$ and $K_{\mathrm{m}}$ of L-isovaline were calculated from data in refs 6 and $25 .{ }^{c}$ Conditions: $100 \mathrm{mM}$ TEA $\cdot$ succinate, $100 \mathrm{mM}$ dipotassium succinate, $50 \mu \mathrm{M}$ PLP, $2 \mathrm{mM}$ AIB, $2 \mathrm{mM}$ sodium pyruvate, $300 \mu \mathrm{M}$ NADPH, 1 unit/mL $2^{\circ} \mathrm{ADH}, 0.1 \mu \mathrm{M}$ DGD, and $0-20 \mathrm{mM}$ inhibitors. ${ }^{d}$ Conditions: same as footnote c except that inhibitors were preincubated with DGD for $30 \mathrm{~min} .{ }^{e} k_{2}$ is the rate constant observed for the restoration of activity from the DGD-AMPP and DGD-s-AEP complexes. Reaction conditions: $100 \mathrm{mM}$ TEA -succinate, $100 \mathrm{mM}$ dipotassium succinate, $50 \mu \mathrm{M}$ PLP, $2 \mathrm{mM}$ sodium pyruvate, 300 $\mu \mathrm{M}$ NADPH, 1 unit/mL $2^{\circ} \mathrm{ADH}, 50,100$, or $200 \mathrm{mM}$ AIB, $0.1 \mu \mathrm{M}$ DGD-AMPP or DGD-s-AEP complex, and $0.16 \mathrm{mM}$ AMPP or $0.04 \mathrm{mM}$ $s$-AEP. Slow binding complexes were prepared by preincubating DGD with AMPP or $s$-AEP for 30 min. Preincubation conditions: $5 \mu$ M DGD, $50 \mathrm{mM}$ TEA-succinate, $50 \mathrm{mM}$ dipotassium succinate, $8 \mathrm{mM}$ AMPP or $2 \mathrm{mM} \mathrm{s}$-AEP, and $50 \mu \mathrm{M}$ PLP. $k_{1}$ was calculated from the equation $K_{\mathrm{i}}{ }^{*}$ $=\left(K_{\mathrm{i}} k_{2}\right) /\left(k_{1}+k_{2}\right) \cdot{ }^{f} k_{1}$ and $k_{2}$ were obtained from spectral kinetic data for the reaction between DGD-PLP and AMPP. Reaction conditions: 2 mM DGD-PLP, $5 \mu \mathrm{M}$ PLP, and $0-35 \mathrm{mM}$ AMPP. ${ }^{g}$ Structurally, this is the closest $\alpha$-aminocarboxylic acid substrate for which kinetic data are available.

the hanging drop vapor diffusion method using a slight modification of the protocol described in ref 11 . Drops of 1 $\mu \mathrm{L}$ containing $30 \mathrm{mg} / \mathrm{mL}$ protein, $5 \mathrm{mM}$ potassium phosphate ( $\mathrm{pH} 7.5), 20 \mu \mathrm{M}$ PLP, and $0.2 \%(\mathrm{w} / \mathrm{v}$ ) sodium azide were mixed with $1 \mu \mathrm{L}$ of reservoir solution containing $15 \%$ PEG4000 (w/v), 15 mM MES-KOH (pH 6.4), 75-200 mM sodium pyruvate, and $20 \mu \mathrm{M}$ PLP, and equilibrated against $1 \mathrm{~mL}$ of this reservoir solution. Crystals generally appeared overnight at room temperature, and grew to a maximum size of $0.3 \mathrm{~mm} \times 0.3 \mathrm{~mm} \times 0.5 \mathrm{~mm}$ after $1-2$ weeks. During the crystallization, microseeding was used. Inhibitors were diffused into DGD crystals by soaking crystals in a stabilizing solution containing inhibitor. For AMPP, the stabilizing solution contained 30\% PEG4000, 15 mM MES-KOH (pH 6.4), $20 \mu \mathrm{M}$ PLP, and $50 \mathrm{mM}$ AMPP. The crystals were soaked in this stabilizing solution for 2 days at room temperature. Since MES is also a ligand of $\operatorname{DGD}(7,12)$, it competes with aminophosphonate binding to DGD. Thus, ACPP or APEP was diffused into crystals soaked in a stabilizing solution without MES. These solutions contained $30 \%$ PEG4000, $40 \mathrm{mM}$ ACPP (pH 6.4) or $40 \mathrm{mM}$ APEP (pH 6.4), $50 \mathrm{mM} \mathrm{KCl}$, and $20 \mu \mathrm{M}$ PLP. Here, ACPP and APEP were used as buffers since their $\mathrm{p} K_{\mathrm{a}}$ values are appropriate. Both isomers of AEP were diffused into DGD crystals by using a stabilizing solution containing $30 \%$ PEG4000, $15 \mathrm{mM}$ Bis-Tris-propane $\cdot \mathrm{HCl}$ (pH 6.4), $30 \mathrm{mM}$ $\mathrm{KCl}, 20 \mu \mathrm{M}$ PLP, and $20 \mathrm{mM} S$-AEP or $20 \mathrm{mM} R$-AEP.

Diffraction data for the DGD-AMPP complex were collected at room temperature, while data for the DGDACPP and DGD-APEP complexes were collected at 100 $\mathrm{K}$ on an RAXIS IV image plate detector using $\mathrm{Cu} \mathrm{K} \alpha$ radiation. DGD- $R$-AEP data were collected on a Bruker CCD detector at $100 \mathrm{~K}$. High-resolution data for the DGD$S$-AEP complex were collected at $100 \mathrm{~K}$ using synchrotron beamline 9-1 at Stanford Synchrotron Radiation Laboratory at a wavelength of $0.98 \AA$ A. Crystals used at $100 \mathrm{~K}$ were harvested from the stabilizing solution described above additionally containing $26 \%$ glycerol and flash-frozen. Reflection intensities were integrated with DENZO and merged using SCALEPACK. The merged data were reduced to structure factors using the combination of SCALEPACK2MTZ, CAD, and TRUNCATE programs from the CCP4 program suite. The structures of DGD inhibitor complexes were determined simply by using the DGD-K structure (PLP, MES ligand, and water molecules

\begin{tabular}{|c|c|c|c|c|}
\hline & AMPP & $\mathrm{ACPP}$ & APEP & $S$-AEP \\
\hline $\begin{array}{l}\text { unit cell dimensions } \\
\text { (A) }(a=b, c)\end{array}$ & $152.8,86.4$ & $151.2,84.8$ & $150.8,84.6$ & $150.8,85.1$ \\
\hline resolution $(\AA)$ & $20.0-2.40$ & $20.0-2.2$ & $20.0-2.6$ & $20.0-2.0$ \\
\hline$R_{\text {sym }}{ }^{a}$ & 0.087 & 0.077 & 0.082 & 0.059 \\
\hline no. of reflections & 23748 & 28332 & 16888 & 38642 \\
\hline redundancy & 9.13 & 3.21 & 3.31 & 6.57 \\
\hline completeness (\%) & 100.0 & 96.1 & 93.8 & 99.2 \\
\hline$R_{\text {cryst }} b(\%)$ & 14.4 & 19.2 & 17.8 & 19.4 \\
\hline no. of protein atoms & 3252 & 3252 & 3252 & 3252 \\
\hline no. of solvent atoms & 168 & 210 & 138 & 235 \\
\hline $\begin{array}{l}\text { no. of heteroatoms } \\
\text { mean } B \text {-factor }\left(\AA^{2}\right)\end{array}$ & 26 & 27 & 30 & 24 \\
\hline entire molecule & 38.2 & 41.6 & 43.3 & 35.4 \\
\hline main chain & 33.6 & 38.1 & 39.5 & 31.6 \\
\hline side chains & 43.5 & 45.6 & 47.6 & 39.7 \\
\hline solvent & 50.9 & 48.9 & 46.5 & 45.2 \\
\hline \multicolumn{5}{|l|}{ rmsd from ideality } \\
\hline bond distances $(\AA)$ & 0.025 & 0.027 & 0.030 & 0.029 \\
\hline bond angles (deg) & 1.78 & 1.94 & 2.07 & 1.98 \\
\hline planar groups $(\AA)$ & 0.016 & 0.012 & 0.015 & 0.016 \\
\hline
\end{tabular}

${ }^{a} R_{\mathrm{sym}}=\sum \sum_{j}\left|I_{j}(h k l)-\langle I(h k l)\rangle\right| / \sum \sum_{j}|I(h k l)|$, where $I_{j}$ is the measured intensity of reflection $j$ and $\langle I\rangle$ is the mean intensity over $j$ reflections. ${ }^{b} R_{\text {cryst }}=\sum|| F_{\text {obs }}(h k l)|-| F_{\text {calc }}(h k l)|| / \sum\left|F_{\text {obs }}(h k l)\right|$, where $F_{\text {obs }}$ and $F_{\text {calc }}$ are the observed and calculated structure factors, respectively. No $\sigma$ cutoff was applied. ${ }^{c}$ The $R_{\text {cryst }}$ and $R_{\text {free }}$ values after CNS refinement were as follows: 17.0 and 20.5\% for AMPP, 21.1 and 24.0\% for ACPP, 17.7 and $22.8 \%$ for APEP, and 20.1 and $22.1 \%$ for $S$-AEP, respectively.

removed) as an initial model for simulated annealing using CNS, since the space group and unit cell parameters were unchanged. During refinement, the program $\mathrm{O}$ was used for model building and electron density interpretation. The final refined structures from CNS were further refined using the TNT refinement package. Only water molecules with $B$ factors of $<85 \AA^{2}$ were retained during the course of the refinement. Data collection and refinement statistics are presented in Table 2.

\section{RESULTS AND DISCUSSION}

The aminophosphonates used in this study (Figure 1) are structural analogues of the natural substrates of DGD (2,2dialkylglycines), in which the reactive carboxylate is substituted with a phosphonate group that is chemically stable in the active site. This allows the determination of the structures of these substrate analogues as Schiff bases with PLP, providing approximations to the structures of the 
reactive external aldimine intermediates with the corresponding amino acid substrates.

Inhibition Kinetics. The inhibition of DGD was studied in detail for AMPP. Figure 2 presents the double-reciprocal plot for AMPP inhibition using the initial linear portions of the progress curves, before the onset of significant slow binding inhibition (see below). The fitted curves show the results of least-squares analysis employing eq 1 . The data strongly indicate that AMPP is, in its initial interaction with DGD, a competitive inhibitor with respect to the AIB substrate. This was expected given the close structural correspondence between the aminophosphonate inhibitor and amino acid substrate. The other aminophosphonate inhibitors were therefore assumed to act by competitive inhibition with respect to AIB. In support of this conclusion, no evidence for amino acid binding to the PMP form of DGD has been found (13), which would open the possibility of noncompetitive or uncompetitive inhibition.

Progress curves for the AIB-pyruvate reaction (Scheme 1) catalyzed by DGD are, in general, not highly linear. This was demonstrated to be due to a slow isomerization of DGDPLP from a more to a less active form (14). The same progress curves in the presence of AMPP were much more nonlinear (Figure 3), and the rate of change of the initial velocity was dependent on inhibitor concentration (data not shown). This suggested that AMPP is a slow binding inhibitor of DGD. Slow binding inhibition has been reviewed in the literature by Morrison and Walsh (10).

Slow binding inhibition by AMPP was confirmed by two other observations. Preincubation of DGD with AMPP for 30 min yields an apparent $K_{\mathrm{i}}$ value (i.e., $K_{\mathrm{i}}^{*}$ ) that is $\sim 10$ fold smaller than that observed without preincubation. A slow regain of activity after the large dilution of the preformed DGD-AMPP complex into a reaction mixture lacking AMPP (Figure 3) and with saturating AIB is also observed.

This latter experiment allowed the determination of $k_{2}$ (Scheme 2), the rate constant for conversion of the final, tight complex to the initial, weak complex, since formation of the initial, weak complex is apparently a rapid equilibrium process and inhibitor dissociation under these conditions is essentially irreversible. The value of $k_{1}$, the rate constant for conversion of the initial, weak complex to the final, tight complex, was calculated from the measured value of $k_{2}$ and the measured $K_{\mathrm{i}}^{*}$ value, using eq 4 . The latter was obtained from the AMPP concentration dependence of the reaction velocities obtained after preincubation for $30 \mathrm{~min}$, using eq 2. The values of these rate constants are reported in Table 1.

The values of $k_{1}$ and $k_{2}$ were measured independently by monitoring directly the reaction of DGD-PLP with AMPP. The PLP in the active site provides a spectroscopic handle on reactions at the active site $(4,15)$. In the case of AMPP, small but readily observable changes in the absorbance spectrum of enzyme-bound PLP were observed after mixing the enzyme and inhibitor (Figure 4). Spectral data were collected as a function of time and analyzed globally using the program SPECFIT. A single-exponential process accounted well for the data, and the apparent rate constants are plotted against total AMPP concentration (i.e., sum of $R$ and $S$ isomers) in the inset of Figure 4. The curve shows the least-squares fit to eq 5 , with $K_{\mathrm{i}}$ being held at its value measured above. The $y$-intercept yields the value of $k_{2}$, while the maximal value is the sum of $k_{1}$ and $k_{2}$. The values of $k_{1}$ and $k_{2}$ obtained from this experiment are reported in Table 1. They agree quite well with the values obtained from steady state kinetic experiments.

The behavior of $S$-AEP was found to be similar to that of AMPP, indicating that it is also a slow binding inhibitor. Similar experiments were thus performed to obtain the values of $K_{\mathrm{i}}, K_{\mathrm{i}}^{*}$, and $k_{1}$ and $k_{2}$. The results of these experiments are reported in Table 1 . The three other inhibitors employed here (APEP, ACPP, and $R$-AEP) showed no signs of slow binding behavior. Their $K_{\mathrm{i}}$ values are reported in Table 1.

Other examples of slow binding inhibitors of PLP dependent enzymes exist in the literature. Atherton et al. (16) first synthesized 1-aminoethanephosphonate as an alanine racemase inhibitor. Walsh's group later demonstrated that it is a slow binding inhibitor of Gram-positive but not Gramnegative bacterial alanine racemases $(17,18)$. It was further demonstrated by NMR and crystallography that it forms a Schiff base with PLP in the active site $(19,20)$, but the structural origin of the slow binding behavior was not clearly elucidated. 1-Aminocyclopropanephosphonate was also shown to be a slow binding inhibitor of alanine racemase, as well as of 1-aminocyclopropanecarboxylate deaminase (21). Other examples of slow binding inhibitors of PLP enzymes that follow a pre-equilibrium association mechanism, i.e., mechanism B of Morrison and Walsh (10), like the aminophosphonates are L-hydrazinosuccinate and L-aminooxysuccinate in their interaction with aspartate aminotransferase $(22,23)$.

Structures of the Aminophosphonate Inhibitor Complexes. Several structures of DGD exist, including those of the MESliganded internal aldimine forms of DGD-Li, DGD-Na, DGD-K, and DGD-Rb $(7,12,24)$, as well as the structures of DGD-K bound to aminocyclopropane-1-carboxylate, phosphopyridoxyl-AIB, and D- and L-cycloserine (8). The four structures of aminophosphonate complexes presented here are also for the DGD-K form. The $R$-AEP complex with DGD diffracted only to low resolution and is thus not reported here. The kinetic data are included for comparison since it is not slow binding but is very similar in structure to $S$-AEP.

Electron density for the four inhibitors bound in the active site is presented in Figure 5. In each case, the inhibitor conformation is unambiguously determined from the electron density, even in the medium-resolution structures (AMPP and APEP).

The superimposed backbone traces of the four inhibitor structures are presented in Figure 6A. Although not included in the figure, the MES-liganded DGD-K backbone trace is indistinguishable from the four structures that are shown. Clearly, as previously concluded on the basis of the other inhibitor structures (8), there is no large-scale conformational change that accompanies substrate analogue binding, as is observed with the prototypical PLP dependent enzyme aspartate aminotransferase.

Figure 6B shows an overlay of the active site structures of the four inhibitor complexes, as well as the MES-liganded internal aldimine form as a reference. Formation of the external aldimine between the inhibitor and PLP requires a rotation of the PLP ring plane forward toward the solvent to prevent steric clashes with K272. The tilt angle observed here for the aminophosphonate inhibitors is $18-23^{\circ}$, which is larger than the value of $13^{\circ}$ observed previously for the 

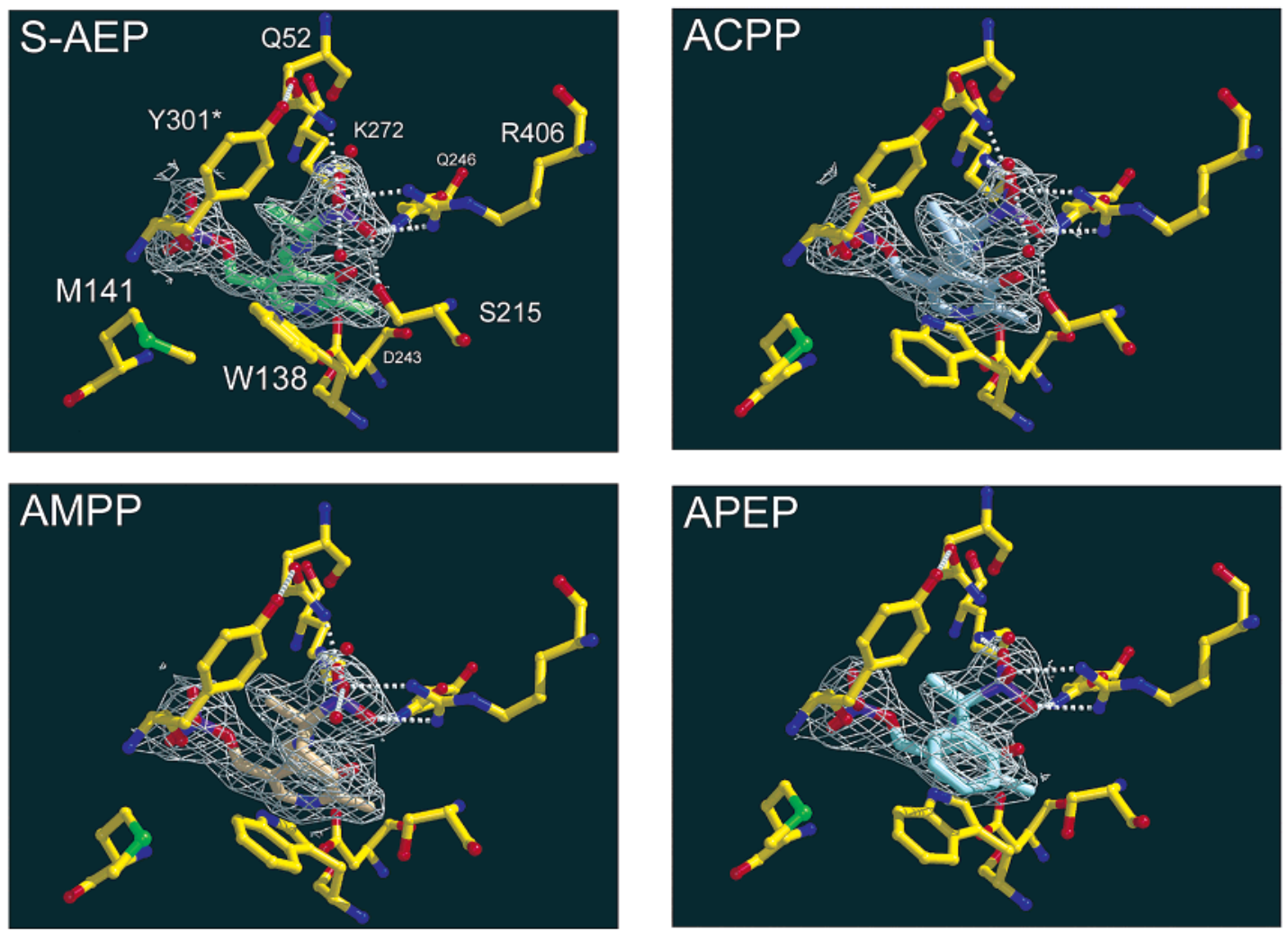

FIGURE 5: Active site structures of the four aminophosphonate inhibitors. The identities are indicated. The $S$-AEP structure has labels for the active site residues. The electron density for the PLP-aminophosphonate Schiff bases is shown contoured at $1 \sigma$. Note the absence of the hydrogen bond between Y310* and Q52 in the ACPP structure. A 3 A cutoff was applied for hydrogen bonds. This figure was created using BOBSCRIPT (26).

aminocyclopropane carboxylate structure (8). Of the four inhibitor structures, only APEP has the PLP-inhibitor Schiff base not planar with the pyridine ring. This is similar to what was found in the aminocyclopropane carboxylate structure. The phenyl ring of APEP stacks tightly with the indole ring of W138 at a distance of $\sim 3 \AA$. The twisting of the Schiff base out of the plane of the pyridine ring (thereby breaking the hydrogen bond between the Schiff base nitrogen and PLP $\mathrm{O}^{\prime}$ ) is apparently required to maintain this minimal van der Waals interaction distance.

The indole ring of W138 changes its angle to accommodate the variations in the size of the inhibitor side chain, with the larger side chains (AMPP, APEP, and ACPP) pushing the benzyl ring of the indole heterocycle toward M141. This causes the M141 side chain to rotate about the $\mathrm{C} \gamma-\mathrm{S} \delta$ bond, moving the terminal methyl group away from W138 to avoid steric clashes.

The edge of the cyclopentyl ring of ACPP is directed toward and in van der Waals contact with Y301*. Accommodation of the large cyclopentyl ring as it is bound requires breaking of the hydrogen bond normally found between Y301* and Q52 (Figure 5). Surprisingly, this hydrogen bond loss does not dramatically lower the affinity of DGD for ACPP, compared to the affinities for the other inhibitors in which it is maintained.

The hydroxyl group of S215 is found in two different positions in the five structures shown in Figure 6B. The variation in the S215 conformation is not correlated with slow binding since the $S$-AEP structure has S215 donating a hydrogen bond to the inhibitor phosphonate group while the AMPP structure has it rotated $145^{\circ}$ such that it hydrogen bonds to a pre-existing water molecule below it. Rather, it appears that the large rotation of S215 is due to the presence of a $\gamma$-carbon on the inhibitor side chain since it occurs in the AMPP and APEP structures, but not in the ACPP structure. This movement of the S215 side chain is required to avoid steric clashes with the inhibitor $\gamma$-carbon.

Yet another variation in structure between the inhibitor complexes occurs near the active site. R251 is located above and behind Q246, which hydrogen bonds to PLP O3' in the MES-liganded structure. This arginine plays no obvious role in the mechanism of either the decarboxylation or transamination reactions. The guanidino group shows two conformations, with concomitant water molecule reorganization, in the four structures presented here (Figure 7A). Neither of these appears to be energetically highly favorable since no counterion interactions are made and hydrogen bonding is minimal. The variation in conformation does not account for slow binding behavior since $S$-AEP and ACPP show one conformation and AMPP and APEP another.

One difference between the inhibitor structures that is correlated with the slow binding behavior of AMPP and $S$-AEP is an extra water molecule located below Met141 in the AMPP and $S$-AEP structures. This water is shown in Figures $6 \mathrm{~B}$ and $7 \mathrm{~B}$ as a large sphere. Figure 7B shows the details of the water binding site. In the AMPP structure, this water apparently forms only one hydrogen bond, to a water just below it in this figure. The $S$-AEP structure shows this water forming two hydrogen bonds, one with the same water as in the AMPP structure and another with R120. The difficulty with assigning this structural difference to the source of slow binding behavior is that there is no clear 

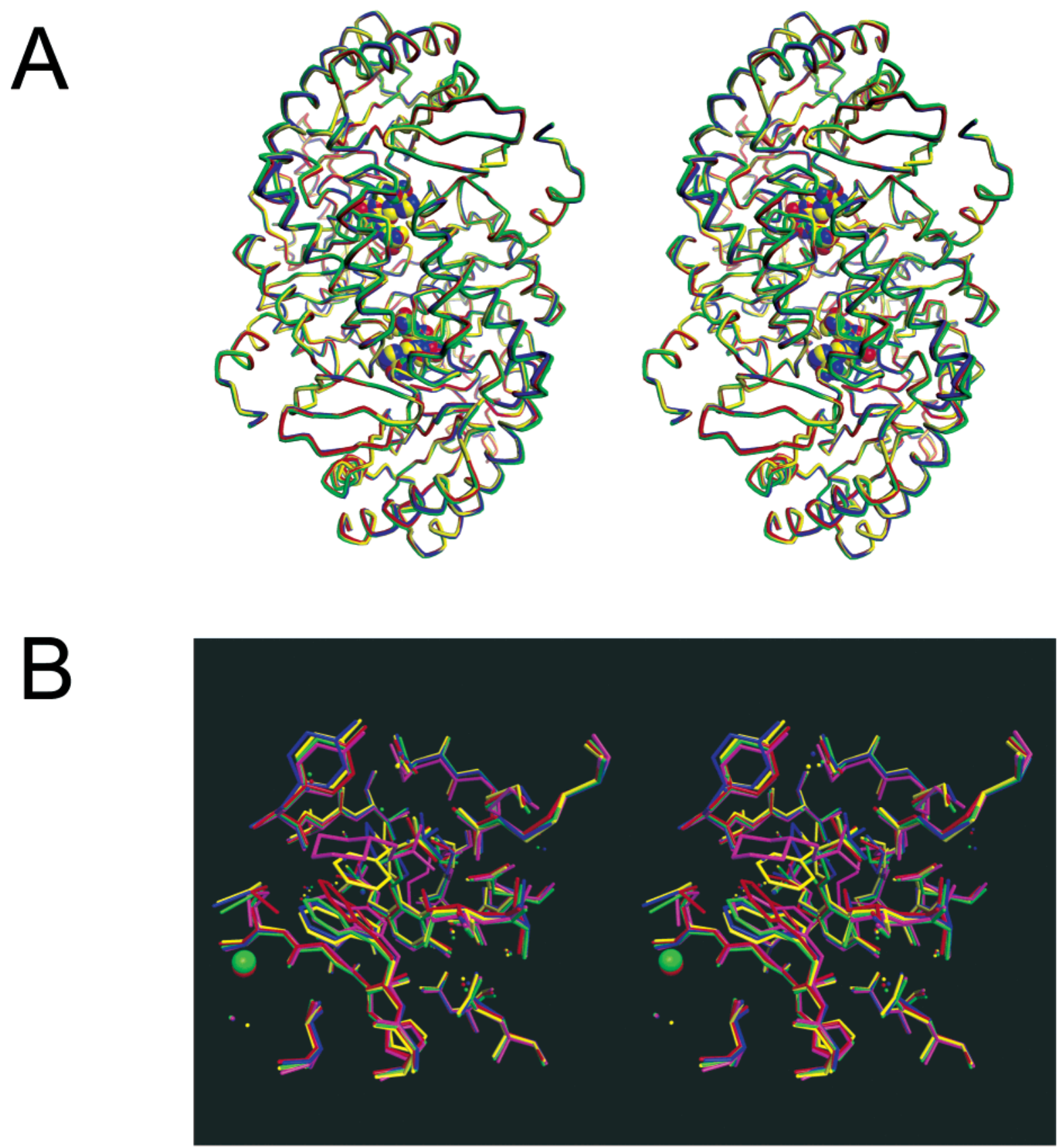

FIGURE 6: Overlay of the $\mathrm{C} \alpha$ traces of the four inhibitor structures (A) and of the active site regions (B). The inhibitors are shown as space filling models in part A. Part B includes DGD-K liganded with MES buffer as a reference. Colors: red for $S$-AEP, green for AMPP, blue for ACPP, yellow for APEP, and pink for MES. The water molecule shown as a large sphere for the AMPP and $S$-AEP structures is unique to slow binding inhibition.

structural explanation for the absence of this water in the ACPP and APEP structures. In the AMPP and $S$-AEP structures, no steric clashes are removed and no extra hydrogen bonds are enabled.

One other structural difference was found to correlate with the slow binding behavior of AMPP and S-AEP. The PLP pyridine nitrogen-D243 OD2 distance is $\sim 2.7 \AA$ for AMPP and $S$-AEP, while it is $\sim 2.9 \AA$ for ACPP and APEP. OD2 of D243 makes two other hydrogen bonding interactions: (1) to the ND1 atom of H139 and (2) to the ND2 atom of N115. These are also found to vary with the pyridine nitrogen-D243 OD2 distance.

Figure 8 presents the distance correlations between the three hydrogen bonding interactions that the OD2 atom of D243 makes for the four phosphonate structures presented here as well as DGD-K, DGD-Na, and the aminocyclopropanecarboxylate inhibitor structures. A monotonic decrease in the H139-D243 interaction distance is observed as the pyridine nitrogen-D243 interaction distance increases. The N115-D243 interaction distance begins to decrease only after the pyridine nitrogen-D243 interaction distance increases beyond $2.8 \AA$. Clearly, there are compensatory changes in hydrogen bonding distances to accommodate the presumed negative charge on D243. The slope of a line fitted to the H139 data in Figure 8 has a slope of $-0.86 \pm 0.08$ when all data are included and $-1.0 \pm 0.1$ when the DGD$\mathrm{Na}$ point is excluded.

The two slow binding phosphonate inhibitors (AMPP and $S$-AEP), with their shorter $\sim 2.7 \AA$ interaction distance between the pyridine nitrogen and D243 OD2, are more 

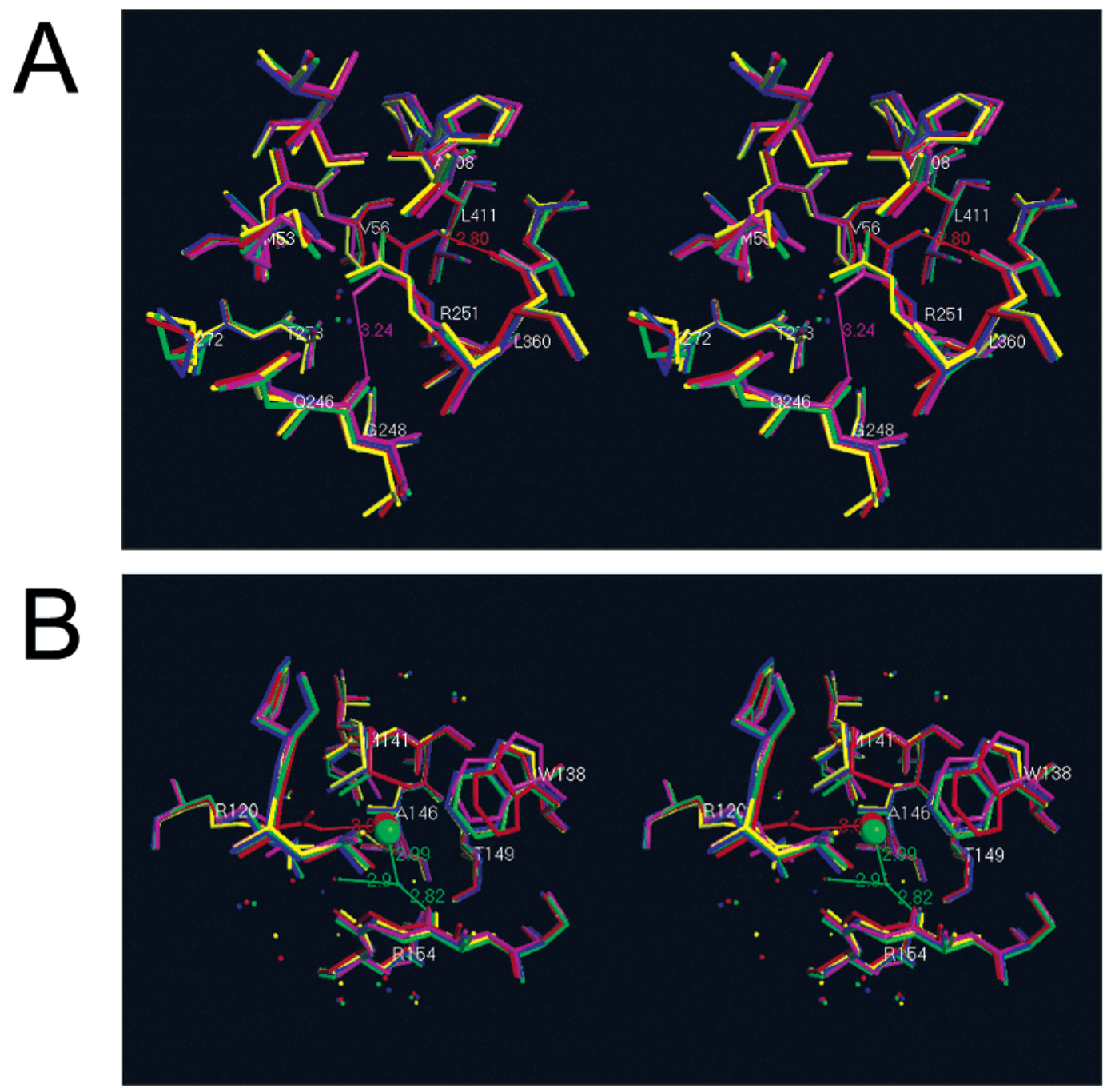

FIGURE 7: (A) Structural variation of R251 with inhibitors and (B) detailed structure of the binding site for the water unique to the AMPP and $S$-AEP active site structures. Colors are the same as in Figure 6.

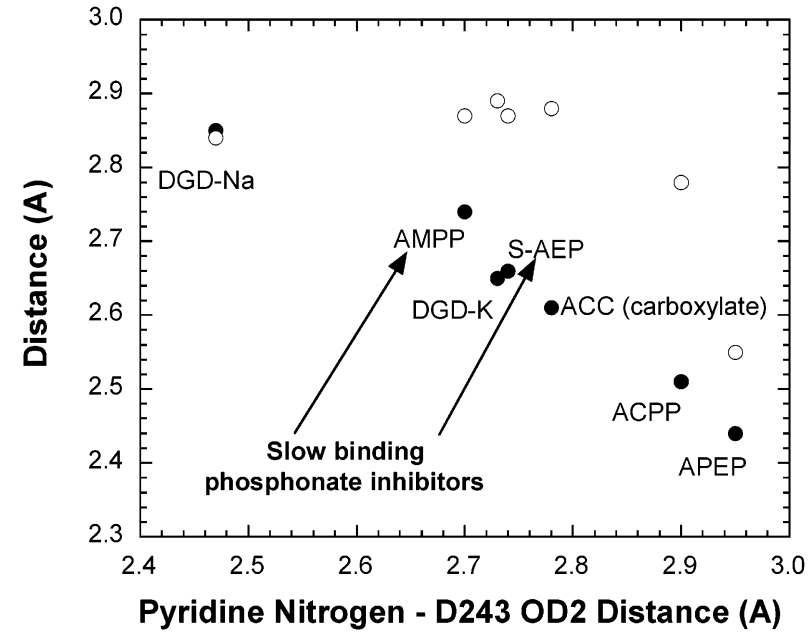

FIGURE 8: Correlation between the PLP pyridine nitrogen-D243 OD2 distance and the D243 OD2-H139 ND1 (-) and D243 OD2N115 ND2 (O) distances. The two slow binding inhibitors are indicated, while ACPP and APEP are not slow binding. ACC is 1-aminocyclopropanecarboxylate.

similar to the DGD-K and aminocyclopropanecarboxylate structures, which presumably have relaxed pyridine nitrogenD243 interactions. The ACPP and APEP structures suggest that shorter pyridine nitrogen-D243 interaction distances are not possible for these due to steric interactions between, mainly, W138 and the larger inhibitor side chains.

Calculations of the energy differences incurred by elongating the pyridine nitrogen-D243 interaction distance were performed with SPARTAN at the 6-31G* level for a simple model in which the methylamine Schiff base of 3-hydroxypyridine-4-aldehyde interacts via the protonated pyridine nitrogen with formate. The geometries of the pyridine nitrogen-formate interaction were constrained to be those found for D243 in the inhibitor structures. These show that the $S$-AEP geometry is $\sim 2 \mathrm{kcal} / \mathrm{mol}$ higher in energy than the AMPP geometry, while the ACPP and APEP geometries are $\sim 6$ and $\sim 9 \mathrm{kcal} / \mathrm{mol}$ higher in energy, respectively. These calculations suffer, of course, from the simplicity of the model since it does not include the H139 and N115 interactions.

Clearly, the variation in the PLP pyridine nitrogen-D243 OD2 interaction distance has the potential to account for the observed $1.4 \mathrm{kcal} / \mathrm{mol}$ difference in binding energy between the rapidly formed DGD-AMPP or DGD-S-AEP complexes and the final, tight binding, isomerized complexes. Thus, it is proposed that the structural basis of the slow binding behavior of AMPP and $S$-AEP is initial formation of a Schiff base between the inhibitor and PLP that resembles 
more the ACPP and APEP structures in their PLP-inhibitor geometries and interactions, followed by slow isomerization to the observed geometries in which the PLP pyridine nitrogen-D243 OD2 interaction distance is shortened. This proposition is supported by the spectra of the initial and final AMPP complexes (obtained from global analysis of the spectral kinetic data, Figure 4) and the ACPP complex. The latter is very similar to the initial AMPP spectrum, while the final AMPP complex shows a red shift of the $\sim 410 \mathrm{~nm}$ band to $\sim 430 \mathrm{~nm}$ and an altered $330 \mathrm{~nm} / 430 \mathrm{~nm}$ ratio, indicative of an altered tautomeric ratio (data not shown). Also, the similarities of the $K_{\mathrm{i}}$ values for all of the inhibitors, which represent rapid, initial complex formation, provide additional support. The observed difference in energy between the initial complex and the final, tight complex is $\sim 1.4 \mathrm{kcal} / \mathrm{mol}$ for both AMPP and $S$-AEP. This probably represents the $6-9 \mathrm{kcal} / \mathrm{mol}$ energy difference due to the variation in the PLP pyridine nitrogen-D243 OD2 interaction distance that is partially but not completely compensated by the covariation in the H139 and N115 interactions with D243.

Implications for Catalysis. Dunathan (5) proposed in 1966 that PLP dependent enzymes can control reaction specificity largely by orienting the bond to be broken such that it is parallel to the $\mathrm{p}$ orbitals of the conjugated Schiff basepyridine ring $\pi$ system. Sun and Toney ( 6 ) provided evidence using alternate substrates that DGD does indeed employ this mechanism for control of reaction specificity. This analysis was based on a functional model of the DGD active site in which there are three binding subsites. The previous carboxylate-containing inhibitor structures (8) show the carboxylate group clearly binding in the so-called "B" subsite, near R406 and S215. The phosphonates, on the other hand, bind between what is ideally considered the A and B subsite, the former placing the substrate carboxylate perpendicular to the pyridine ring plane through hydrogen bonding with Q52 and K272 (7). This is probably the result of the steric bulk of the three phosphonate oxygens and the requirement to compensate electrostatically its double negative charge versus the single charge of a substrate carboxylate, since the "in between" site allows interaction with Q52, K272, Q246, R406, and (for $S$-AEP and ACPP) S215.

This suboptimal representation by the aminophosphonates of the substrate bound in the A subsite notwithstanding, the AMPP, S-AEP, and ACPP structures show an enticing correlation of aldimine structure with the reactivities of their corresponding substrates. APEP is excluded from this analysis since its Schiff base is not coplanar with the pyridine ring. The $\mathrm{C} 4^{\prime}-\mathrm{N}-\mathrm{C} \alpha-\mathrm{P}$ dihedral angle is $-139^{\circ}$ for AMPP, $-159^{\circ}$ for $S$-AEP, and $-161^{\circ}$ for ACPP, which correspond to $k_{\text {cat }}$ values for the analogous substrates of $14,0.2$, and $0.98 \mathrm{~s}^{-1}$, respectively (Table 1 ). Thus, the inhibitor with the $\mathrm{C}-\mathrm{P}$ bond closest to being parallel to the p orbitals of the $\pi$ system corresponds to the substrate with the highest $k_{\text {cat }}$ value. This suggests that the structural features of the phosphonate inhibitors that determine binding geometry also affect the binding geometry of the corresponding carboxylate substrates, which in turn determines reactivity through stereoelectronic effects.

\section{ACKNOWLEDGMENT}

Portions of this research were carried out at the Stanford Synchrotron Radiation Laboratory, a national user facility operated by Stanford University on behalf of the U.S. Department of Energy, Office of Basic Energy Sciences. The SSRL Structural Molecular Biology Program is supported by the Department of Energy, Office of Biological and Environmental Research, and by the National Institutes of Health, the National Center for Research Resources, the Biomedical Technology Program, and the National Institute of General Medical Sciences.

\section{REFERENCES}

1. John, R. A. (1995) Biochim. Biophys. Acta 1248, 81-96.

2. Jansonius, J. N. (1998) Curr. Opin. Struct. Biol. 8, 759-769.

3. Mehta, P. K., and Christen, P. (2000) Adv. Enzymol. Relat. Areas Mol. Biol. 74, 129-184.

4. Zhou, X., Jin, X., Medhekar, R., Chen, X., Dieckmann, T., and Toney, M. D. (2001) Biochemistry 40, 1367-1377.

5. Dunathan, H. C. (1966) Proc. Natl. Acad. Sci. U.S.A. 55, 712716.

6. Sun, S., Zabinski, R. F., and Toney, M. D. (1998) Biochemistry $37,3865-3875$.

7. Toney, M. D., Hohenester, E., Keller, J. W., and Jansonius, J. N. (1995) J. Mol. Biol. 245, 151-179.

8. Malashkevich, V. N., Strop, P., Keller, J. W., Jansonius, J. N., and Toney, M. D. (1999) J. Mol. Biol. 294, 193-200.

9. Soroka, M. (1990) Liebigs Ann. Chem., 331-334.

10. Morrison, J. F., and Walsh, C. T. (1988) Adv. Enzymol. Relat. Areas Mol. Biol. 61, 201-301.

11. Toney, M. D., Keller, J. W., Pauptit, R. A., Jaeger, J., Wise, M. K., Sauder, U., and Jansonius, J. N. (1991) J. Mol. Biol. 222, $873-875$.

12. Toney, M. D., Hohenester, E., Cowan, S. W., and Jansonius, J. N. (1993) Science 261, 756-759.

13. Zhou, X., and Toney, M. D. (1999) Biochemistry 38, 311-320.

14. Zhou, X., Kay, S., and Toney, M. D. (1998) Biochemistry 37, 5761-5769.

15. Christen, P., and Metzler, D. E. (1985) Transaminases, Wiley, New York.

16. Atherton, F. R., Hall, M. J., Hassall, C. H., Lambert, R. W., Lloyd, W. J., and Ringrose, P. S. (1979) Antimicrob. Agents Chemother. $15,696-705$.

17. Badet, B., and Walsh, C. (1985) Biochemistry 24, 1333-1341.

18. Badet, B., Inagaki, K., Soda, K., and Walsh, C. T. (1986) Biochemistry 25, 3275-3282.

19. Copie, V., Faraci, W. S., Walsh, C. T., and Griffin, R. G. (1988) Biochemistry 27, 4966-4970.

20. Stamper, G. F., Morollo, A. A., and Ringe, D. (1999) Biochemistry $38,6714$.

21. Erion, M. D., and Walsh, C. T. (1987) Biochemistry 26, 34173425.

22. Yamada, R., Wakabayashi, Y., Iwashima, A., and Hasegawa, T. (1986) Biochim. Biophys. Acta 871, 279-284.

23. Scaman, C. H., Palcic, M. M., McPhalen, C., Gore, M. P., Lam, L. K., and Vederas, J. C. (1991) J. Biol. Chem. 266, 5525-5533.

24. Hohenester, E., Keller, J. W., and Jansonius, J. N. (1994) Biochemistry 33, 13561-13570.

25. Snell, E. E. (1968) Pyridoxal catalysis: enzymes and model systems, pp 479-490, Interscience Publishers, New York.

26. Esnouf, R. M. (1999) Acta Crystallogr. D55 (Part 4), 938-940. BI026318G 